\title{
Combined 18-FDG-PET/Contrast-Enhanced CT in the Diagnosis of Recurrent Colorectal Cancer: A Prospective Monocentric Pilot Study
}

\author{
Tannoury J 1,5, Mule $\mathbf{S}^{2,3}$, Itti $\mathbf{E}^{2,3}$, Luciani $\mathbf{A}^{2,3}$, \\ Rotkopf $\mathbf{H}^{1,5}$ and Sobhani $\mathrm{I}^{1,5 *}$ \\ ${ }^{1}$ Department of Gastroenterology, Henri Mondor \\ University Hospital, France \\ ${ }^{2}$ Department of Medical Imaging and Nuclear medicine, \\ Henri Mondor University Hospital, France \\ ${ }^{3}$ Department of Radiology, Henri Mondor University \\ Hospital, France \\ ${ }^{4}$ Department of Oncology, Creteil, Henri Mondor \\ University Hospital, France \\ ${ }^{5}$ EC2M3-EA7375 Paris EST Creteil University (UPEC), \\ France \\ *Corresponding author: Iradj Sobhani, Department \\ of Gastroenterology, Henri Mondor University Hospital, \\ Gastroentérologie, 51 Av Marechal de Lattre de Tassigny, \\ 94010 Creteil, France
}

Received: February 16, 2021; Accepted: March 22, 2021; Published: March 29, 2021

\begin{abstract}
Contrast-enhanced CT scan and 18DG-PET are used in the staging and surveillance of Colorectal Cancer (CRC). We here evaluate the feasibility and performances of systematically combining 18-FDG-PET and contrastenhanced CT whether as a stand-alone diagnostic examination, or as 2 separate examinations, for the early detection of recurrent colorectal cancer. 45 patients underwent 159 planned diagnostic follow-up sessions: 109 (68\%) were performed as a combined modality during the same examination and 50 were performed as two separate examinations and acquired at different timepoints. Fourteen patients experienced recurrence during the study period. The sensitivity of 18-FDG-PET and contrast-enhanced CT scan in detecting a recurrent disease was $84.4 \%$ and $86.6 \%$ respectively with a specificity of $96 \%$ and $97 \%$ respectively. 18-FDG-PET and contrast-enhanced CT findings' were concordant in $79 \%$ of cases. The overall sensitivity and specificity in detecting CRC recurrence were $80 \%$ and $99 \%$ respectively in the combined procedure and $89 \%$ and $98 \%$ respectively in the two-time procedure. The positive predictive value of the procedure (combined or two-time procedure) for the detection of recurrent CRC was $100 \%$ with, however a shorter delay for decision with the combined [7.7 vs. 12.2 days] $(p<0.05)$.
\end{abstract}

The combined procedure is feasible in almost $2 / 3$ of cases. When compared to the two-time procedure, it is performant in detecting recurrent CRC with shorter delay to therapy.

Keywords: PET; CT; 18-FDG; Colon; Cancer

\section{Key Points}

- Combined 18-FDG-PET / contrast-enhanced CT in one diagnostic session has been previously shown as an innovative and cost-effective imaging tool in the monitoring strategy of aggressive lymphoma

- Herein, we report a pilot study in the surveillance of colon and rectal cancer patients (stage III or IV) after curative surgery as an accurate one-time procedure

- Tts performant in detecting recurrences with shorter delay to therapy is shown and speculation on costs and acceptance discussed.

\section{Introduction}

Mortality rates from Colorectal Cancer (CRC) have declined significantly in the last years [1]. This improvement is attributed, at least in part, to recent development in diagnostic imaging modalities. Contrast-enhanced Computed Tomography (CT) is recognized as an effective tool for diagnosis, staging and monitoring of CRC [2]. In the last years, fluorodeoxyglucose positron emission tomography (18-FDG-PET) has been increasingly used for assessment of early recurrence and therapy response monitoring. Indeed, our group has reported the first study on the early detection of CRC by using 18-FDG-PET as a survey tool [3]. More recently, metabolic response shown by 18-FDG-PET has been suggested to be a more useful exam as compared to contrast-enhanced CT for detecting early recurrences in various diseases [4]. However, in a recent open-label multicentre trial, adding routine 6-monthly 18-FDG-PET increased costs without decreasing treatment failure rates in patients in remission of CRC (stage II perforated, stage III, or stage IV) [5]. Furthermore, in a recent update of a Cochrane Review, there were no effect on overall survival of intensifying the follow-up programs of patients after curative surgery for colorectal cancer [6]. We suggest that the combination of imaging information from multiple modalities in a single procedure may offer a promising tool for prompt clinical diagnosis and therapeutic applications. Combined 18-FDG-PET/ contrast-enhanced CT in one diagnostic session has been shown as an innovative and cost-effective imaging tool in the monitoring strategy of aggressive lymphoma [7] while studies in CRC are still lacking. The aim of our study was to assess the performance and the technical feasibility of this combined procedure in detecting early recurrences compared with 18-FDG-PET and contrast-enhanced CT performed as separate acquisitions at different times.

\section{Patients and Methods}

From 2008 to 2014, in the area of CRETEIL, 45 patients with high-risk CRC recurrence treated and followed up in a 3 -yr period in two public hospitals (i.e. the University Hospital Henri Mondor
Austin J Radiol - Volume 8 Issue 3 - 2021

ISSN : 2473-0637 | www.austinpublishing group.com

Sobhani et al. (C) All rights are reserved
Citation: Tannoury J, Mule S, Itti E, Luciani A, Rotkopf H and Sobhani I. Combined 18-FDG-PET/ContrastEnhanced CT in the Diagnosis of Recurrent Colorectal Cancer: A Prospective Monocentric Pilot Study. Austin J Radiol. 2021; 8(3): 1128. 
and Intercommunal de Creteil-CHIC) were enrolled in the study (Table 1). They were all assigned to have a combined procedure and were prospectively evaluated currently for feasibility, performances and results. The study protocol was approved by the institutional ethics committee (Comite de Protection des Personnes, protocol $n$ PP 13-043) (CPP 07-035; revised Jan. 2008 and April 2012; National Ministry Register 2007-AO1138-45) and patients provided written informed consent before study inclusion. High risk recurrence during a 3-year follow-up was defined as stage II CRC with tumor perforation, stage III CRC or stage IV CRC with complete resection of all synchronous and metachronous metastases with or without neo adjuvant therapy. All patients were routinely assessed prospectively at regular 3-monthly intervals up to 36 months after curative surgery, or until death. All patients had to be scheduled for either a single combined or a two-time procedure 18-FDG-PET/contrast-enhanced CT every 6 months during the follow-up period. When conditions of the combined procedure were not met (mostly for logistical reasons), patients underwent 18-FDG-PET then a contrast-enhanced CT as separate acquisitions at different times (different days) with results analyzed independently. Body contrast-enhanced CT systematically included CT acquisition covering the cervical region to the pelvic groin, which was initiated $80 \mathrm{~s}$ after the injection of $1.5 \mathrm{cc} / \mathrm{kg}$ of contrast agent. Post-processing reconstructions were performed on native data in the transverse plane to yield at least $2.5 \mathrm{~mm}$ thick sections for image analysis. All acquisitions were performed using multi-slice CT scanner. Body 18DG-PET was performed on fasting patients $(\geq 6 \mathrm{~h})$ controlled by glycaemia $<2 \mathrm{~g} / \mathrm{L}, 60 \mathrm{~min}$ after IV injection of $4-5 \mathrm{MBq} /$ $\mathrm{kg}$ 18DG. Examinations consisted of a low-dose CT followed by an emission scan in 9-11 steps from cervical to pelvic regions; they were reconstructed without and with attenuation correction by using iterative algorithms for SUV computation.

When contrast-enhanced CT and 18-FDG-PET were performed as a single examination (where contrast-enhanced CT scan was performed immediately after completion of PET acquisition, planned with the same scout view, and on the same device), 18DG was infused first and CT contrast agent was injected after the scanning of 18-FDGPET was completed. All readers had access to the patient's clinical history. A senior nuclear medicine specialist and a senior radiologist reviewed 18-FDG-PET and CECT images, respectively, and classified the recurrence status as yes, no or doubtful. In the evaluation of 18-FDG-PET, a lesion was considered positive whenever it showed a non-physiological increase of FDG uptake.

The primary outcome was the detection of a recurrent disease or death. All images were reviewed during the MDT meetings to confirm the recurrence or the remission and consequently to decide of the upcoming treatment strategy. Thus, the performance of the different imaging modalities in the detection of a recurrent disease (true positive) and the confirmation of a remission (true negative) was referred to the MDT decision. Recurrent diseases were validated either by histopathology or by clinical-radiological assessment in multidisciplinary team (MDT) meetings. We performed a comparative analysis of procedures performed as initially planned - i.e. combined 18-FDG-PET/contrast enhanced CT during the same session versus the two examinations performed separately. The feasibility rate of the combined procedure was determined and the mean delay to a formal diagnosis of relapse was calculated in the two groups.

\section{Results}

All 45 patients enrolled underwent 159 diagnostic procedures during the study period, including 109 as a combined 18-FDG-PET /contrast-enhanced CT and 50 consisting of two separate exams (18-FDG-PET and contrast-enhanced CT as separate acquisitions at different times). Thus, the feasibility of the combined procedure in routine was $68 \%$. The combined procedure was missed because of missing appointments or lack of recent renal function tests. According to the MDT's decision, the sensitivity of 18-FDG-PET scan and contrast-enhanced CT scan in detecting a recurrent disease was $84.4 \%$ and $86.6 \%$ respectively with a specificity of $96 \%$ and $97 \%$ respectively (Table 2 ). The overall concordance rate between 18-FDG-PET and contrast-enhanced CT findings was 79\% (126/159 procedures). A total of 88 concordant findings were identified in the combined modality group $(\mathrm{n}=109,80 \%)$ and 38 in the two-time procedure group $(\mathrm{n}=50,76 \%)$. False positive 18-FDG-PET results $(\mathrm{n}=11)$ were mainly due to increased $18-\mathrm{FDG}$ uptake in the bowel whereas false positive contrast-enhanced CT results $(n=6)$ were related to non-specific post-operative peritoneal adhesions $(n=3$,

Table 1: Main characteristics of patients enrolled.

\begin{tabular}{|c|c|}
\hline \multirow{2}{*}{ Patients' characteristics } & Number of patients N=45 (\%) \\
\hline \multirow{2}{*}{ Gender } & Men: $20(44.4)$ \\
\cline { 2 - 2 } & Women: $25(55.6)$ \\
\hline \multirow{2}{*}{ Disease stage at inclusion } & II: $6(13.3)$ \\
\cline { 2 - 2 } Tumor localization & III: $22(48.9)$ \\
\cline { 2 - 2 } & IV: $17(37.8)$ \\
\hline \multirow{2}{*}{ neoadjuvant therapy } & Colon: $33(73.3)$ \\
\hline \multirow{2}{*}{ adjuvant therapy } & Rectum: $12(26.7)$ \\
\hline & Yes: $18(40)$ \\
\hline
\end{tabular}

Table 2: Performance of 18-FDG-PET and IV CT scan in detecting CRC recurrence.

\begin{tabular}{|c|c|c|c|}
\hline \multicolumn{2}{|c|}{ 18DG-PET (n=159) } & \multicolumn{2}{c|}{ Contrast-enhanced CT $(\mathbf{n = 1 5 9 )}$} \\
\hline $\begin{array}{c}\text { Positive } \\
\text { lesions }\end{array}$ & $\begin{array}{c}\text { Confirmed } \\
\text { recurrence }\end{array}$ & $\begin{array}{c}\text { Positive } \\
\text { lesions }\end{array}$ & $\begin{array}{c}\text { Confirmed } \\
\text { recurrence }\end{array}$ \\
\hline $38 / 159$ & $27 / 38$ & $32 / 159$ & $26 / 32$ \\
\hline Negative PET & $\begin{array}{c}\text { Confirmed } \\
\text { recurrence }\end{array}$ & Negative CT & $\begin{array}{c}\text { Confirmed } \\
\text { recurrence }\end{array}$ \\
\hline $121 / 159$ & $5 / 121$ & $127 / 159$ & $4 / 127$ \\
\hline
\end{tabular}

Table 3: Performance of separated and combined FDG-PET/ IV CT scan in detecting $C R C$ recurrence.

\begin{tabular}{|c|c|c|}
\hline & \multicolumn{2}{|c|}{ Procedure } \\
\hline & $\begin{array}{l}\text { FDG-PETI IV CT one acquisition } \\
\qquad(n=109)\end{array}$ & $\begin{array}{l}\text { FDG-PETI IV CT separate } \\
\text { acquisitions }(n=50)\end{array}$ \\
\hline & \multicolumn{2}{|c|}{ Performance in the diagnosis of recurrent CRC (\%) } \\
\hline Se & 80 & 89 \\
\hline Sp & 99 & 98 \\
\hline PPV & 100 & 100 \\
\hline NPV & 99 & 97 \\
\hline
\end{tabular}

Se: Sensitivity; Sp: Specificity; PPV: Positive Predictive Value; NPV: Negative Predictive Value IV CT: Contrast-Enhanced CT. 
$50 \%)$, two false-positive lung lesion and one hepatic lesion. We observed 5/121 (4.13\%) false negative results with 18-FDG-PET and 4/127 (3.14\%) with contrast-enhanced CT. All false negative findings were finally detected after combining the two exams. According to the MDT criteria, fourteen patients (31\%) had recurrent disease at the end of the study period. The overall sensitivity and specificity of the imaging in detecting CRC recurrence was $80 \%$ and $99 \%$ respectively in the combined procedure and $89 \%$ and $98 \%$ respectively in the two-time procedure. When 18-FDG-PET and contrast-enhanced CT scans were both positive, recurrent disease was always confirmed (positive predictive value of $100 \%$ ). The median delay between the first imaging study and the multidisciplinary staff decision was 7.7 days [range 1-16 days] with the combined procedure and 12.2 days [range 2-22 days] with the two-time procedure $(\mathrm{p}<0.05)$.

\section{Discussion}

Here we evaluate the feasibility and the performance of a combined 18-FDG-PET/contrast-enhanced CT procedure for both metabolic and morphological assessment of CRC recurrence in a series of 45 patients with high risk of recurrence. This procedure appears feasible in close to $70 \%$ of cases in the course of routine care. There was no discrepancy in all 14 patients with documented recurrence. Hence, combining IV injection to PET/CT with the implemented technical protocol does not impede the individual performance of each modality.

Despite the efficiency of both 18-FDG-PET and contrastenhanced CT in detecting CRC metastases, 3\%-15\% of recurrences may be missed by each of these procedures when performed alone and the respective sensitivity and specificity of both techniques is not significantly different. Interestingly however, false negative findings on either technique appear to be detected on the other one. In addition, contrast-enhanced CT may be limited by kidney failure impeding the IV injection of contrast media, and 18-FDG-PET may be limited in detecting liver metastasis because of low SUV due to chemotherapy or mucous component of the primary tumor [8]. These biases cannot always be avoided in the routine practice, although in the current study all patients had normal renal function tests and have ended their chemotherapy before entering the follow up period. The value of combining 18-FDG-PET to contrast enhanced CT in the routine surveillance of CRC remains controversial. In a recent openlabel multicentre trial, we showed that monitoring CRC recurrence with 18-FDG-PET added every 6 months increased costs without decreasing treatment failure rates in patients in remission of CRC [5]. Thus, we suggested that 18-FDG-PET should be limited to selected patients such as those with stage IV CRC who should undergo curative surgery or those with rise of blood tumor marker. Since the fused anatomical images from CT and functional images from PET have been shown relevant for better detection of distant metastases and/or recurrence after curative surgery [9], we currently suggest a single hybrid imaging including two exams as a valuable alternative.

The benefits of the combination of 18-FDG-PET and contrastenhanced CT over contrast-enhanced CT alone and unenhanced 18-FDG-PET have been well established for the monitoring of abdominal and pelvic malignant diseases [10] and lymphoma [11]. A retrospective study performed in 53 patients showed that contrastenhanced 18-FDG-PET/CT was superior to non-enhanced 18-FDG-
PET/CT for exact definition of regional nodal status in rectal cancer [12]. When another study conducted on 100 patients with various malignant diseases showed that contrast-enhanced CT provides additional value for patient therapy management as compared to low-dose non-contrast CT in 18-FDG-PET/CT protocols [13].

In our study, abnormal images as detected by both metabolic and anatomical imaging were always associated with recurrent disease. That is to notice that misdiagnosis or delay in making medical decision observed with 18-FDG-PET and contrast-enhanced CT performed as two separate exams was mostly related to false positive 18-FDG-PET results $(n=7)$ and to additional exams implied by doubtful contrastenhanced CT images. Although the diagnostic delay may not be clinically significant, the two-time procedure led to additional exams and thus additional cost, increased patient compliance and radiation exposure. Indeed, as shown in our previous prospective study [5] including a medical cost evaluation, performing two exams enhances significantly the global cost per patient. In the current study, patients had one or more exams during the follow up period. This means in a scenario only two procedures were performed per year, each patient would receive double ionization radiation dosage, would lose two working day with doubling fees due to the transportation. We acknowledge that the major limitation of our study is the absence of abdominal MRI, which is now considered the preeminent imaging modality for the characterization, and detection of liver metastases. However, surveillance is rarely performed for liver metastasis alone and MRI is limited in the evaluation of extrahepatic disease, particularly the lungs, hence the current preference for CT over MRI. In conclusion, although both modalities provide similar sensitivity and specificity figures for the detection of tumor recurrence in treated CRC patients, 18-FDG-PET/contrast-enhanced CT as a combined exam can provide morphologic and functional data in a single session reducing additional diagnostic imaging exams. The availability of combined contrast-enhanced CT images and 18-FDG-PET images improves diagnosis of colorectal cancer recurrence. The combined procedure often leads to prompt therapy decision with probably lower costs compared to the two-time procedure. Patient's clinical pathway should certainly be reviewed to increase the feasibility of this protocol in order to identify individuals most likely to benefit from this imaging approach.

\section{Statements}

\section{Statement of ethics}

All patients were informed that anonymized data from their medical files could be used for medical research purposes and that they were free to refuse such use of the data in accordance with French legislation.

\section{Disclosure statement}

Iradj Sobhani has received financial support for travel expenses, registration at international conventions, or research from Biocodex, Novartis, IPSEN, and Pfizer.

Alain Luciani has received financial support for participation to research board and training program, from Bracco Group and General Electric Healthcare.

\section{Funding sources}

No funding from the pharmaceutical industry was received for 
this study.

Academic sources from EC2M3-EA7375 UPEC.

\section{Author contributions}

Iradj Sobhani and Alain Luciani contributed to design and conduct the study.

Sébastien Mulé, Emmanuel Itti, Hugo Rotkopf, contributed to the analysis and editing Jenny Tannoury took the lead in writing the manuscript.

All authors contributed to analyses the data and critically revised the manuscript for important intellectual content.

\section{References}

1. Ansa BE. Evaluation of Colorectal Cancer Incidence Trends in the United States (2000-2014). J Clin Med. 2018; 7: 22

2. Tan $\mathrm{CH}$, lyer R. Use of computed tomography in the management of colorectal cancer. World J Radiol. 2010; 2: 151-158.

3. Mondor $\mathrm{H}$, Lattre $\mathrm{M}$. Early detection of recurrence by 18 FDG-PET in the follow-up of patients with colorectal cancer. 2008; 98: 875-880.

4. Kitajima K, Nakajo M, Kaida $\mathrm{H}$, Minamimoto R. Present and future roles of FDG-PET/CT imaging in the management of gastrointestinal cancer : an update. 2017; 79: 527-543

5. Sobhani I, Itti E, Luciani A, Baumgaertner I, Layese R, André T, et al. Colorectal Cancer (CRC) monitoring by 6-monthly 18-FDG-PET/CT: an open-label multicentre randomised trial. Ann Oncol. 2018; 29: 931-937.
6. Jeffery M, Hickey BE, Hider PN. Follow-up strategies for patients treated for non-metastatic colorectal cancer. Cochrane database Syst Rev. 2019; 9 : CD002200.

7. Haioun C, Itti E, Rahmouni A, Brice P, Rain J-D, Belhadj K, et al. [18F] fluoro2-deoxy-D-glucose positron emission tomography (FDG-PET) in aggressive lymphoma: an early prognostic tool for predicting patient outcome. Blood. 2005; 106: 1376-1381.

8. Van Kessel CS, Buckens CFM, Van Den Bosch MAAJ, Van Leeuwen MS Van Hillegersberg R, Verkooijen HM. Preoperative imaging of colorectal liver metastases after neoadjuvant chemotherapy: A meta-analysis. Ann Surg Oncol. 2012; 19: 2805-2813.

9. Zhu A, Lee D, Shim H. Metabolic positron emission tomography imaging in cancer detection and therapy response. Semin Oncol. 2011; 38: 55-69.

10. Trial RC, Otero HJ, Yap JT, Patak MA, Johnston CJ, Hj O, et al. Evaluation of low-density neutral oral contrast material in PET/CT for tumor imaging: results of a randomized clinical trial. AJR Am J Roentgenol. 2009; 193: 326 332.

11. D'souza MM, Jaimini A, Bansal A, Tripathi M, Sharma R, Mondal A, et al FDG-PET/CT in lymphoma. Indian J Radiol Imaging. 2013; 23: 354-365.

12. Tateishi U, Maeda T, Morimoto T, Miyake M, Arai Y, Kim EE. Non-enhanced CT versus contrast-enhanced $\mathrm{CT}$ in integrated $\mathrm{PET/CT}$ studies for nodal staging of rectal cancer. Eur J Nucl Med Mol Imaging. 2007; 34: 1627-1634.

13. Pfannenberg AC, Aschoff $P$, Brechtel $K$, Müller $M$, Klein M, Bares R, et al. Value of contrast-enhanced multiphase CT in combined PET/CT protocols for oncological imaging. Br J Radiol. 2007; 80: 437-445. 